\title{
TRIZ APLICADO AO PROCESSO DE TREFILAÇÃO*
}

Guery Augusto Echeverria Martínez ${ }^{1}$ Gustavo Aristides Santana Martínez

\section{Resumo}

O profissional brasileiro tem uma grande capacidade em criar soluções. $\mathrm{Na}$ área de trefilação o resultado desta criatividade é facilmente encontrado em diversos pontos do processo. No entanto esta capacidade não segue um roteiro, simples ou complexo, de tal forma a possibilitar respostas inovativas em alto nível e por toda a equipe da organização. Este trabalho propõe o ensino da metodologia TRIZ e de ferramentas auxiliares a todos os colaboradores das trefilarias. A TRIZ é uma metodologia sistemática, isto é baseado em regras, o que permite a obtenção de uma solução direcionada, portanto, muito mais assertiva no processo de inovação. A natural criatividade do profissional brasileiro pode ser amplificada com o aprendizado de uma metodologia que sistematize o desenvolvimento de novas soluções para antigos e novos problemas no processo de trefilação. $O$ aprendizado das ferramentas básicas de TRIZ, associado a outras ferramentas, podem tornar os profissionais da área de trefilação mais confiantes e efetivos na análise de problemas, geração de ideias e prevenção de falhas.

Palavras-chave: Trefilação; TRIZ; Inovação; Criatividade; Ensino.

\section{Abstract}

\section{TRIZ APPLIED TO WIRE DRAWING PROCESS}

The Brazilian professional has a great ability to create solutions. In the drawing area the result of this creativity is easily found in various parts of the process. However, this ability does not follow a script, simple or complex, so as to enable innovative solutions at a high level and by the entire organization staff. This work proposes the teaching of the TRIZ methodology and of auxiliary tools to all drawing plants employees. TRIZ is a systematic methodology, i.e., based on rules, which allows obtaining a targeted solution, therefore, much more assertive in the innovation process. The natural creativity of the Brazilian professional can be amplified with the learning of a new methodology that systematizes the development of new solutions for old and new problems in drawing process. The learning of the TRIZ basic tools, associated to other tools, can make professionals from the drawing area more confident and effective in problem analysis, ideas generation and failures prevention. Keywords: Wire drawing; TRIZ; Innovation; Creativity. 


\section{INTRODUÇÃO}

O brasileiro tem demonstrado grande capacidade criativa de forma natural, o que tem gerado profissionais inovativos nas mais diversas áreas de atuação. Não é diferente na área de conformação plástica dos materiais, mais especificamente na área de trefilação de fios, barras e tubos metálicos.

Soluções as mais criativas possíveis podem ser encontradas nas trefilarias do Brasil. Desde o uso de pequeno pedaço de arame enrolado em torno do arame que está sendo trefilado a seco, com o objetivo de evitar a formação de tuneis de sabão que levam a redução do carreamento do lubrificante a interface fio fieira, até modificações as mais complexas e inimagináveis.

Este simples exemplo demonstra a capacidade do profissional brasileiro em pensar e resolver problemas em todo os níveis de atuação profissional.

Esta capacidade pode ser amplificada com o aprendizado de uma metodologia que sistematize a criatividade em propor e desenvolver novas soluções para antigos e novos problemas no processo de trefilação.

Este artigo propõe o ensino de ferramentas da metodologia Teoria de Solução Inventiva de Problemas (TRIZ) aos colaboradores das trefilarias com o objetivo de contribuir na análise de problemas, geração de ideias e prevenção de falhas.

\subsection{Revisão Bibliográfica}

A trefilação é um processo de conformação plástica dos metais, geralmente realizada a frio, no qual uma barra, um fio ou um tubo, devidamente limpo e coberto com um lubrificante, é tracionado através de uma ferramenta dura, resistente ao desgaste e com uma abertura cônica, chamada de fieira, onde sofre uma redução da secção transversal. Este processo é uma interação complexa de vários parâmetros definidos por:

- Propriedades do fio (limite de escoamento, módulo de elasticidade, taxa de deformação, encruamento);

- Lubrificante (coeficiente de atrito, viscosidade, tratamento superficial do fio) e;

- Geometria da fieira (ângulo de redução, comprimento da região de calibração, redução em área, material).

Na figura 1 é mostrada a simulação da tensão radial no fio em três pontos distintos, isto é, na primeira camada (nó 580) em camada intermediaria (nó 315) e na camada central (nó 50) do fio à medida que passa pelas fieiras com 10 e 18 graus.

Por se tratar de um processo complexo, os problemas estão constantemente presentes desde a entrada da matéria prima, e materiais auxiliares, até a saída do produto acabado. Esta complexidade exige um alto nível de comprometimento, por parte de todos os colaboradores das trefilarias, em termos de criatividade e inovação. No entanto somente boa vontade, sem o uso de métodos de criatividade e inovação, não é suficiente.

Existem mais de 190 métodos de criatividade conhecidos que podem ser aplicados a área de trefilação. A grande diversidade de métodos criativos existentes possui uma origem em comum que pode ser derivada em apenas dois tipos de classes: Métodos Intuitivos (estudos psicológicos e de tentativa e erro) e Métodos Sistemáticos (baseados em passos estruturados). 


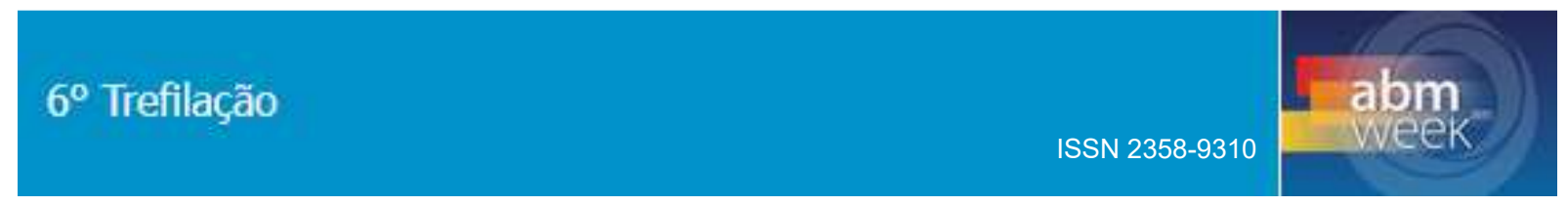



a) $2 \beta=10^{\circ}$

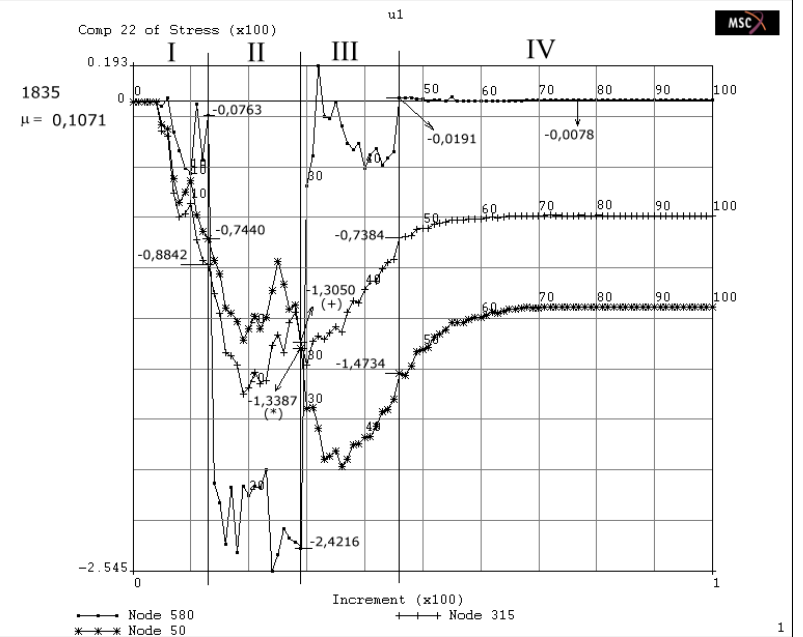

b) $2 \beta=18^{\circ}$

Figura 1 - Comportamento da tensão radial no fio durante o processo.

Em seu trabalho, CHANGQING; HUANG e MA $\{1\}$, compararam as metodologias de inovação com TRIZ e concluíram que, mesmo depois de 50 anos de existência, a TRIZ ainda é amplamente utilizada no mundo.

TRIZ é um acrônimo russo de "teorijarezhenijaizobreta-telskih zadach", que significa "Teoria de Solução Inventiva de Problemas" e em inglês \{2\}, é conhecido também como TIPS (Theory of Inventive Problem Solving). Desenvolvida pelo engenheiro, inventor, cientista, jornalista e escritor, Genrich Saulovich Altshuller (1926-1998) e seus colaboradores, a TRIZ começou na Rússia nos anos 50 para auxiliar os inventores russos em seus problemas técnicos. Hoje, possui muitos praticantes no mundo inteiro, mas a maioria ainda está presente em países da antiga URSS, visto que a difusão da TRIZ no Ocidente iniciou-se apenas nos anos $90\{3\}$.

Segundo os aspectos da análise a TRIZ é mais prática do que outros métodos de inovação $\{1\}$. De certo, a TRIZ, por ser um método orientado, mostra-se capaz de gerar soluções poderosas. O seu poder de inovação, é baseado em regras, o que permite a obtenção de uma solução direcionada, portanto, muito mais precisa. É possível notar também, que, comparativamente, a TRIZ é a metodologia mais sistemática que as outras e o suporte base de conhecimento é um fator muito importante no processo de inovação. A fundamentação prévia das patentes é um importante instrumento competitivo e fonte de informação tecnológica $\{4\}$.

Ao generalizar e agrupar as soluções repetidamente utilizadas nas criações, desenvolvimentos e melhorias das patentes, foram extraídos os Princípios Inventivos, que são heurísticas ou sugestões de soluções para determinados problemas. Existem duas maneiras de se utilizar os 40 Princípios Inventivos: a primeira, seria observando diretamente os princípios a fim de obter uma sugestão para a solução do problema diretamente (ou seja, fazer um Checklist), e a segunda maneira, seria utilizar as contradições presentes nos Parâmetros de Engenharia (melhoramentos $\mathrm{X}$ agravamento). Cada um dos princípios são grandezas que podem servir de orientação para o resultado final de determinado problema.

Ao contrário dos métodos tradicionais de criatividade (brainstorming, tentativa e erro...), o objetivo da TRIZ é tentar erradicar as contradições. Uma das maneiras de se resolver as contradições (Figura 2), é utilizando a Matriz das Contradições (melhoramentos $\mathrm{X}$ agravamento). E para utilização da matriz, deve-se estabelecer as seguintes relações: Parâmetro a ser melhorado, Parâmetro de agravamento. 


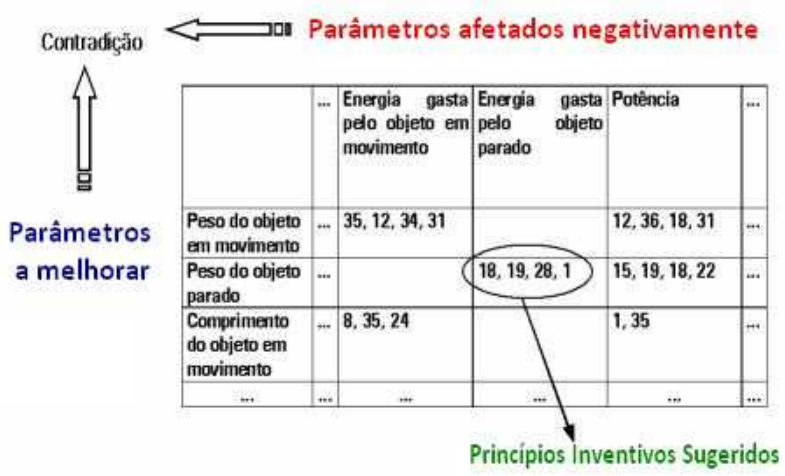

Figura 2: Matriz de Contradição $\{3\}$

Antes mesmo de utilizar uma das ferramentas para iniciar a resolução das contradições, é necessária uma análise prévia da situação problema. A identificação do problema é primordial e há alguns instrumentos para realizá-la. Os Instrumentos de Análise do problema podem ser: ISQ (Innovation Situation Questionair), Formulação do problema, Análise Substância-Campo, etc. $\{5\}$. Diante de um panorama geral, a identificação/descrição do problema deve responder algumas questões fundamentais e conter, sumariamente, algumas características principais: ambiente operacional para o problema, requisitos de recursos, função utilitária primária, efeitos prejudiciais, resultado ideal para o problema $\{6\}$.

A análise do problema possibilita descrevê-lo e enfatizá-lo. É necessário transformar uma "situação inicial" em um "problema específico". Sendo a "situação inicial" aquela que necessita algum aperfeiçoamento, ou seja, a melhora de alguma característica não desejável. $O$ instrumento ISQ permite que estas necessidades sejam levantadas, e também, realiza a descrição do problema. Para XIMENEZ $\{7\}$ é aconselhável a sua utilização pois é composto por perguntas estratégicas que possibilita a obtenção do maior número de informações relevantes possíveis e assim, ajudar a definir o problema corretamente.

Acreditamos que o aprendizado das ferramentas básicas de TRIZ, associado a outras ferramentas, podem tornar as pessoas mais confiantes e efetivas na análise de problemas, geração de ideias e prevenção de falhas.

\section{MÉTODO PROPOSTO}

A presente proposta assume como premissa verdadeira que todos os problemas de uma organização devem ser resolvidos de forma conjunta pois são do tipo administrativo, técnico ou físico. Deste ponto de vista as respostas precisam ocorrer no nível operacional, tático e estratégico da organização. Isto significa dizer que todos os colaboradores da empresa devem ser convocados a participarem do treinamento na metodologia TRIZ associada a ferramentas auxiliares.

Neste trabalho entendemos como ferramentas auxiliares todo e qualquer instrumento de interesse na formação dos treinandos verificado pela particularidade da empresa onde esta proposta está em curso. Como exemplo de ferramentas auxiliares podemos dar o Brainstorm, PBL, 5W2H, SWOT, ISQ, etc.

Embora a peculiaridade da empresa, onde irá ocorrer a implantação deste método, tenha vital importância acreditamos que grupos de trabalho de no máximo 10 pessoas devem ser formados. É de suma importância que cada grupo tenha colaboradores das mais diferentes áreas da organização. 
$\mathrm{Na}$ primeira etapa será realizado um levantamento das condições técnicas e administrativas da organização. Considerando que uma organização, mesmo que do mesmo ramo de atuação, possui características diferentes nesta etapa será desenhada a forma de conduzir as etapas seguintes. Uso somente da matriz de contradições, campo substância ou ARIZ são definidas levando-se em consideração o levantamento das condições técnicas e administrativas da organização. Este primeiro trabalho auxilia também na definição das ferramentas auxiliares a serem aplicadas.

A segunda etapa é constituída de reuniões presenciais, de no máximo três horas, onde a metodologia TRIZ e as ferramentas auxiliares deverão ser ensinadas. Esta etapa precisará de 20 a 30 horas de dedicação.

$\mathrm{Na}$ terceira etapa temos o aprofundamento individual do conhecimento na metodologia e irá ocorrer a distância. Esta etapa precisará de 30 a 40 horas de dedicação on-line.

$\mathrm{Na}$ etapa de aprofundamento os colaboradores devem entrar em contato com soluções práticas genéricas oferecidas pela metodologia, assim como casos mais próximos com a área de trefilação. Um bom exemplo, de uso de ferramentas auxiliares a metodologia TRIZ, e o uso da Taylor-PBL nesta etapa. A construção do algoritmo para diagnosticar as situações de problema, a transposição da inercia psicológica e o efetivo desenvolvimento da abstração é objeto desta etapa.

A segunda e terceira etapas têm como objetivo o entendimento da importância da metodologia TRIZ no desenvolvimento da capacidade em identificar problemas e resolver de forma criativa com a utilização dos recursos disponíveis.

$\mathrm{Na}$ quarta última e permanente etapa será necessário a formação dos grupos de discussão, aplicação e acompanhamento das análises de problemas, geração de ideias e prevenção de falhas levantadas. Esta etapa tem como finalidade vencer em definitivo a inércia psicológica e extrair o máximo da capacidade criativa e inovativa dos colaboradores de forma sistemática.

\section{RESULTADOS ESPERADOS}

Esta proposta pretende obter resultados de criatividade do segundo nível de inventividade, minimamente. Isto significa afirmar que em mais de $80 \%$ dos treinandos serão capazes de utilizar soluções conhecidas para solucionar problemas e, os outros $20 \%$ serão capazes de desenvolver novas ideias para solucionar problemas.

Pessoas mais confiantes e efetivas na análise de problemas, geração de ideias e prevenção de falhas são um dos resultados a serem atingidos com esta proposta.

Trefilarias mais inovativas pela aplicação de uma forma sistemática do processo de inovação também é objeto desta metodologia.

\section{CONCLUSÃO}

A estratégia teórico-metodológica apresentada neste trabalho e uma discussão preliminar para a aplicação de TRIZ e ferramentas auxiliares na solução de problemas na área de trefilação. A correta aplicação das três primeiras etapas e a garantia da manutenção da quarta etapa podem aumentar significativamente a capacidade dos colaboradores na análise de problemas, geração de ideias e prevenção de falhas no processo de trefilação de fios, barras e tubos. 


\section{REFERÊNCIAS}

1 CHANGQING, Gao, HUANG Kezheng, and MA Fei. Comparison of innovation methodologies and TRIZ. The TRIZ Journal, Issue (September) (2005).

2 DEMARQUE, E. TRIZ: Teoria para a Resolução de Problemas Inventivos aplicada ao Planejamento de Processos na indústria automotiva. 2005. 160 f.Trabalho de curso (Mestrado Profissionalizante em Engenharia Automotiva) Escola Politécnica da Universidade de São Paulo, São Paulo, 2005.

3 CARVALHO, M. A. Modelo Prescritivo para a Solução Criativa de Problemas nas Etapas Iniciais do Desenvolvimento de Produtos. Dissertação de Mestrado. Programa de Pós-graduação em Engenharia de Produção. Orientador: Nelson Back. UFSC, 1999.

4 FERREIRA, Ademir Antônio; GUIMARÃES, Edílson Rodrigues; CONTADOR, José Celso. Patente como instrumento competitivo e como fonte de informação tecnológica. Gest Prod, v. 16, n. 2, p. 209-21, 2009.

5 JAFARI ,Mostafa; AKHAVAN, Peyman; REZA , Hamid Zarghami; ASGARI, Naser Exploring the effectiveness of inventive principles of TRIZ on developing researchers' innovative capabilities, Journal of Manufacturing Technology Management, Vol. 24 Iss 5 pp. $747-767,2013$.

6 INNOSKILLS - Guide to Support Innovation in Small and Middle Enterprises (SMEs)-EU Programme Leonardo da Vinci), 2014.

7 XIMENES, Robert Dias. Estudo e aplicação da metodologia TRIZ: desenvolvimento de um projeto conceitual para escolha de mancais para cabeçote de uma máquina de ultraprecisão. Tese de Doutorado. Universidade de São Paulo, 2011. 